Editorial

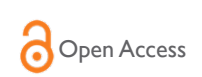

CrossMark

\title{
Implant hygiene and patient compliance
}

\section{Editorial}

According to the American Academy of Implant Dentistry, 3 million Americans have implants with an estimated 500,000 to be placed annually. Ten percent of U.S. dentists currently place implants. ${ }^{1}$ Success rates of dental implants have been reported in scientific literature to be over $90 \%$. While this is an impressive rate, it may underestimate the number of patients who develop implant problems such as peri-mucositis and peri-implantitis. In one study involving 212 patients with implants, the prevalence of peri-mucositis and peri-implantitis was reported to be $64.6 \%$ and $8.9 \%$, respectively. ${ }^{2}$ Based upon a multi-variate analysis, poor plaque control was one of the factors that were associated with increased odds for having periimplant disease. In another study, a high proportion of implants with a diagnosis of peri-implantitis were associated with no accessibility/ capability for appropriate oral hygiene measures, while accessibility for hygiene to the implant was rarely associated with peri-implantitis. ${ }^{3}$ These data suggest that with good oral hygiene education with the right oral care "tools" can help control plaque and help lessen the likelihood of developing peri-implant conditions.

Is patient hygiene controllable? I think it is time for dental professionals to have a reality check for patients who promise to maintain their implant prosthesis with proper hygiene but in reality, this is rarely done according to our recommendations. I think we need to understand that "this is a reality". Even when we instruct our patients it is extremely difficult for us to monitor their proper hygiene especially around the fixed implant prosthesis. Speaking with the patient about hygiene sometimes seems to be a bit boring however it's extremely important. While we, as dentists, may not have time to reinforce this, our dental assistants and hygienists can be very effective in communicating and instruction of proper oral hygiene.

It is a fact of life that patients will not remember our instructions. We must imprint in their minds that is their responsibility to maintain proper hygiene in order to protect their implant prosthesis and their investment. Well there is a way to monitor a patient utilizing the new technology in power brushes. Specifically we use in our office the Oral-B Genius power brush which has a specific oscillating-rotatingpulsating motion, tracking technology of daily brushing, a sensor to prevent excessive pressure, a feature via use of an app that allows the patient to visually see areas that were missed. The Oral-B OscillatingRotating-Pulsating Power Toothbrush lines are currently the only powered toothbrushes to receive the ADA Seal of Acceptance.

Since we are aware that many patients rarely follow our guidelines in maintaining their implants then it is incumbent upon dental professionals to take the next step. With the patients permission we can actually track the brushing from their new Genius brush. This can then be brought in on their next appointment for us to review. By spending a small amount of extra time, if we take the extra step in helping our patients help themselves, we're doing the right thing and have diminished our liability from failure to communicate proper hygiene to the patient.
Volume 8 Issue 3 - 2017

\author{
Joseph Massad \\ Department of Dentistry, USA
}

Correspondence: Joseph Massad, Department of Dentistry, USA, Email joe@joemassad.com

Received: September 26, 2017 | Published: October 03, 2017

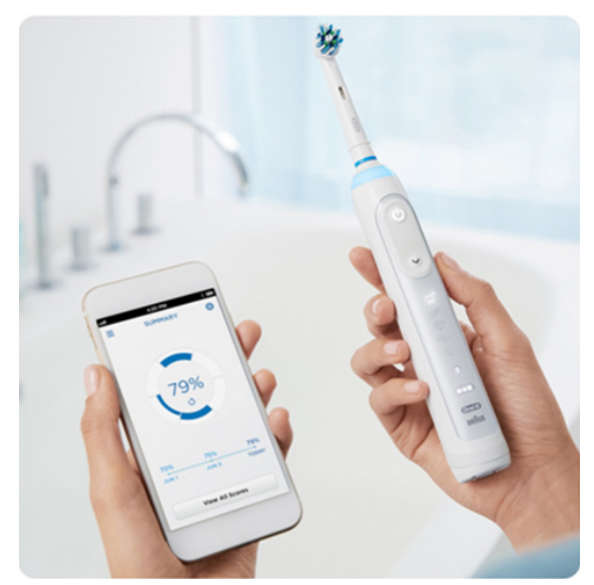

Acknowledgments

None.

\section{Conflicts of interest}

The authors declare that there is no conflict of interest.

\section{Funding}

None.

\section{References}

1. American Academy of Implant Dentistry, USA; 2017.

2. Ferreira SD, Silva GL, Cortelli JR, et al. Prevalence and riskvariables for peri-implant disease in Brazilian subjects. $J$ Clin Periodontol. 2006;33(12):929-935.

3. Serino G, Strom C. Peri-implantitis in partially edentulous patients: association with inadequate plaque control. Clin Oral Implants Res. 2009;20(2):169-174. 